\section{BMJ Open} Ophthalmology

\title{
MRI-based 3D retinal shape determination
}

\author{
Luc van Vught (D) , ${ }^{1,2}$ Denis P Shamonin (D) , ${ }^{3}$ Gregorius P M Luyten (D) ,' \\ Berend C Stoel (D) , Jan-Willem M Beenakker (1) ${ }^{1,2}$
}

To cite: van Vught $L$, Shamonin DP, Luyten GPM, et al. MRI-based 3D retinal shape determination. BMJ Open Ophthalmology 2021;6:e000855. doi:10.1136/ bmjophth-2021-000855

- Additional supplemental material is published online only. To view, please visit the journal online (http://dx.doi. org/10.1136/bmjophth-202100085).

Received 16 July 2021 Accepted 30 October 2021

Check for updates

\section{(C) Author(s) (or their} employer(s)) 2021. Re-use permitted under CC BY-NC. No commercial re-use. See rights and permissions. Published by BMJ.

${ }^{1}$ Department of Ophthalmology, Leiden University Medical Center, Leiden, The Netherlands ${ }^{2}$ Department of Radiology, CJ Gorter Center for High Field MRI, Leiden University Medical Center, Leiden, The Netherlands ${ }^{3}$ Department of Radiology, Division of Image Processing (LKEB), Leiden University Medical Center, Leiden, The Netherlands

Correspondence to Luc van Vught; I.van_vught@ lumc.nl

\section{ABSTRACT}

Objective To establish a good method to determine the retinal shape from MRI using three-dimensional (3D) ellipsoids as well as evaluate its reproducibility. Methods and analysis The left eyes of 31 volunteers were imaged using high-resolution ocular MRI. The 3D MR-images were segmented and ellipsoids were fitted to the resulting contours. The dependency of the resulting ellipsoid parameters on the evaluated fraction of the retinal contour was assessed by fitting ellipsoids to 41 different fractions. Furthermore, the reproducibility of the complete procedure was evaluated in four subjects. Finally, a comparison with conventional two-dimensional (2D) methods was made.

Results The mean distance between the fitted ellipsoids and the segmented retinal contour was $0.03 \pm 0.01 \mathrm{~mm}$ $($ mean $\pm S D)$ for the central retina and $0.13 \pm 0.03 \mathrm{~mm}$ for the peripheral retina. For the central retina, the resulting ellipsoid radii were $12.9 \pm 0.9,13.7 \pm 1.5$ and $12.2 \pm 1.2 \mathrm{~mm}$ along the horizontal, vertical and central axes. For the peripheral retina, these radii decreased to $11.9 \pm 0.6$, $11.6 \pm 0.4$ and $10.4 \pm 0.7 \mathrm{~mm}$, which was accompanied by a mean $1.8 \mathrm{~mm}$ posterior shift of the ellipsoid centre. The reproducibility of the ellipsoid fitting was $0.3 \pm 1.2 \mathrm{~mm}$ for the central retina and $0.0 \pm 0.1 \mathrm{~mm}$ for the peripheral retina When 2D methods were used to fit the peripheral retina, the fitted radii differed a mean $0.1 \pm 0.1 \mathrm{~mm}$ from the $3 \mathrm{D}$ method.

Conclusion An accurate and reproducible determination of the $3 \mathrm{D}$ retinal shape based on MRI is provided together with $2 \mathrm{D}$ alternatives, enabling wider use of this method in the field of ophthalmology.

\section{INTRODUCTION}

Measurements of the geometrical shape of the eye have obtained an important position within the field of ophthalmology. Biometry and corneal topography measurements, for example, have shown to be important for the diagnosis, treatment and follow-up of patients with refractive conditions. ${ }^{12}$ Additionally, such measurements have been used to create and analyse personalised models of the eye in research settings. ${ }^{3-6}$ Thus far, these geometrical assessments have mainly focused on the anterior segment of the eye, and the three-dimensional shape of the retina is often disregarded. This three-dimensional shape, however, is suggested to be of clinical relevance for peripheral vision and ocular radiotherapy planning. ${ }^{7-9}$

\section{Key messages}

What is already known about this subject?

MR-imaging can be used to quantify the retinal shape in terms of ellipsoidal radii.

\section{What are the new findings?}

The derived retinal shape, expressed as ellipsoid, is highly dependent on the evaluated fraction of the retina.MR-imaging is a reproducible method to quantify the three-dimensional retinal shape.

\section{How might these results change the focus of research or clinical practice?}

These results, together with the full description of the entire method, provide an accurate method to determine the three-dimensional retinal shape, enabling subject-specific retinal shape evaluation in other ophthalmic research fields, such as myopia, peripheral vision and ocular radiotherapy.

Several assessments of the retinal shape have already been performed using off-axis laser interferometry. ${ }^{10-13}$ These evaluations were, however, limited to measuring the visual field up to $35^{\circ}$ in the horizontal and vertical meridian, covering the central $70^{\circ}$ of the visual field and thus only the more central retina. ${ }^{11} 12$ The use of MRI as an alternative to off-axis interferometry has also been proposed, since it is not limited by the optical opacity of tissues and can therefore provide a three-dimensional (3D) evaluation of the complete eye. ${ }^{7} 1415$ These 3D MR-images enable direct modelling of the retina as an ellipsoid, but were initially hindered by their limited resolution. Improvements in MRI techniques have enabled acquisition of three dimensional images of the eye with a higher, sub-millimetre, resolution, ${ }^{16-18}$ enabling the determination of topographic maps of the complete retinal contour, ${ }^{17}$ and increasing the accuracy of the determined ellipsoid descriptions.

However, multiple methodological choices, such as the location of the ellipsoid centre and the definition of an optical or central axis, potentially affect the resulting ellipsoidal model. Unfortunately, the exact effect of these choices on the ellipsoid parameters is not known, hampering comparisons 


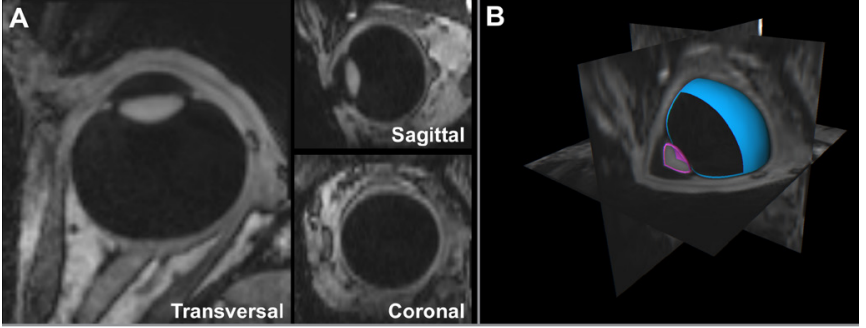

C

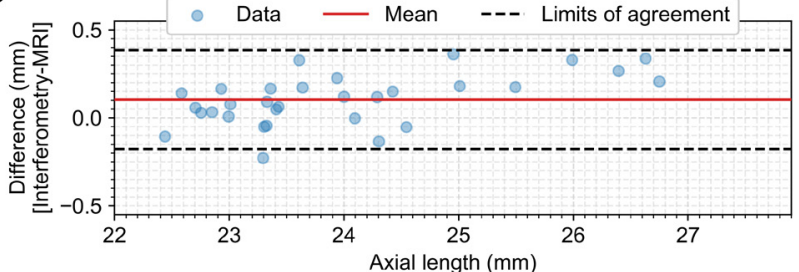

Figure $1 \mathrm{MRI}$ acquisition and automated segmentation results. (A) A transversal, sagittal and coronal reformat of the same 3D acquisition. (B) The result of the segmentation of this MR-images. (C) The agreement between MRI and laser interferometry in distance between the lens centre and inner limiting membrane of the retina. The mean difference between the measurements was $0.10 \mathrm{~mm}$, with BlandAltman limits of agreement of -0.18 and $0.39 \mathrm{~mm}$. 3D, threedimensional.

between different studies. Additionally, even though an earlier study showed good agreement between MRI and biometry-based axial length determinations, ${ }^{17}$ the reproducibility and accuracy of an MR-based retinal shape determination has not been determined. These insights are required to further implement retinal shape determination techniques in ophthalmic research and clinical care. Therefore, we assessed the accuracy, comparability and reproducibility of MRI-based 3D retinal shape determination to provide a basis for further incorporation of this method in ophthalmic research and therewith move towards clinical implementations.

\section{MATERIAL AND METHODS}

The left eyes of 31 volunteers without prior ocular surgery were examined at the Leiden University Medical Center. Patients were not involved in the design of this study. Written informed consent was obtained from all subjects prior to enrolment in the study. Subjects were required to have no contraindications for MRI-scanning.

\section{Measurements}

Ocular MRI was performed on a Philips Achieva 7 Tesla whole-body magnet (Philips, Best, The Netherlands) using a custom-made receive eye coil and a volume transmit coil (Nova Medical, Wilmington, Massachusetts, USA). ${ }^{17} 19$ T1-weighted MR-images were acquired using a 3D inversion recovery turbo gradient echo technique (figure $1 \mathrm{~A}$ ). The scan had a spatial acquisition resolution of $0.5 \times 0.5 \times 1.0 \mathrm{~mm}^{3}$, a field of view of $46 \times 46 \times 38 \mathrm{~mm}^{3}$, a total scan time of slightly less than $3 \mathrm{~min}$, an inversion time of $1280 \mathrm{~ms}$ and a shot interval of $3 \mathrm{~s}$. The repetition time, echo time and flip angle were $2.5 \mathrm{~ms}, 4.55 \mathrm{~ms}$ and $16^{\circ}$, respectively. A cued-blinking protocol was used to minimise eye-motion artefacts. ${ }^{1920}$ In addition to the MRI measurement, the axial length of the eye from cornea to inner limiting membrane was measured using laser interferometry (Lenstar LS 900; Haag-Streit AG, Koeniz, Switzerland; mean of two scans).

\section{Segmentation}

The retinal contour, defined as the boundary between the hypointense vitreous and the hyperintense retinasclera complex, was automatically segmented on the T1-weighted MR-images using in-house-developed software based on the MevisLab image-processing platform (V2.5.1, Fraunhofer MeVis, Bremen, Germany), as has been previously described. ${ }^{1921}$ In short, the outer borders of the lens and vitreous body were automatically detected using a subdivision fitting algorithm. ${ }^{21} 22$ This algorithm uses intensity-based cost functions to delineate the lens and vitreous body with subvoxel precision and returns 3D meshes of the lens and retina (figure 1B). The algorithm requires an initial guess as a starting point, which was automatically derived from the MR-images using a combination of intensity-based labelling and region growing, noise reduction, anisotropic diffusion filtering, multithresholding and morphological operations, as described by Beenakker et al. ${ }^{17}$ Prior to the subdivision fitting, all initial guesses were inspected and manually corrected if necessary.

\section{Eye orientation}

Due to the positioning within the MRI-scanner, the subjects' gazing direction does not correspond to any axis of the acquired MR-image. Moreover, the fovea and the associated visual axis are not visible on MRI. ${ }^{17}$ As a result, the MR-derived retinal shape data cannot be compared between subjects or with other ophthalmic measurements without defining a common axis. Therefore, a central axis was defined as the line between the centre of the segmented lens and the centre of the segmented vitreous body. ${ }^{17}$ This central axis was subsequently aligned with the anterior-posterior axis by rotating the eye around the centre of the lens.

In vivo, such eye rotations are accompanied by cyclotorsion, a rotation around the visual axis of which both the magnitude and direction are related to the change in ocular gazing direction. ${ }^{23-25}$ Similarly, the alignment of the MR-data with a common axis will also induce a rotation around the central axis, ${ }^{24}$ and the magnitude of this induced rotation will likely be affected by chosen alignment method. To evaluate the relevance of this potential variation in induced rotation, three different alignment methods were applied and compared. In the first method, two consecutive rotations were performed in a head-fixed coordinate system. In the second method, two consecutive rotations were performed in an eye-fixed coordinate system, where the second rotation axis is affected by the first rotation. In the third method, only one rotation was performed around the axis defined by Euler's theorem. 
A visual and mathematical description of these methods can be found in online supplemental appendix A.

After these rotations, the principal axes of the MR-data correspond to the horizontal, vertical and central axes. All three methods will thus result in the same gazedirection, but potentially with different amounts of cyclotorsion. The differences between the retinal shapes resulting from three rotation methods were evaluated by comparing the resulting cyclotorsion and calculating the mean distance between the three retinal shapes using k-dimensional trees. ${ }^{26}$

\section{Agreement between MRI and laser interferometry}

To evaluate the accuracy of the boundary definition of the automatic segmentation of the retinal contour, a comparison with the axial length measured using laser interferometry was performed. As the presence of air in front the eye and under the eyelids can locally affect MRI and therewith potentially the shape of the cornea on the resulting images, the cornea was not segmented. Therefore, an adjusted axial length, defined as the distance from the centre of the lens to the inner limiting membrane of the retina, was used to compare laser interferometry and MRI. ${ }^{17}$

\section{Retinal shape determination}

Retinal shapes were quantified by fitting a 3D ellipsoid to the segmented retinal contour points after correcting for gazing differences. The 3D ellipsoid was defined as:

$$
\frac{h^{2}}{R_{h}}+\frac{v^{2}}{R_{v}}+\frac{c^{2}}{R_{c}}=1,
$$

where $h, v$ and $c$ are the coordinates of the contour points in the horizontal, vertical and central axis, and $R_{h}, R_{v}$ and $R_{c}$ are the radii of the ellipsoid along its principal axes. These contour points were expressed relative to the centre coordinates of the ellipsoid, $\left(C_{h}, C_{v}, C_{c}\right)$, and the principle axes of the ellipsoid could be angulated around this centre by angles $\alpha, \beta$ and $\gamma$. The ellipsoid radii, centre coordinates and angulations provided nine df to the fitting algorithm.

The fitting algorithm consisted of three phases, being the generation of an initial guess, the determination of the centre coordinates and the determination of the radii and angulation. To ensure that the fitting algorithm was not biased or dependent on the coordinate transformations, a geometric fit rather than an algebraic fit was performed. ${ }^{27} \mathrm{~A}$ detailed description of the complete fitting algorithm can be found in online supplemental appendix A. The correspondence between each fitted ellipsoid and the retinal contour points was defined as the mean of the absolute shortest 3D distances between the retinal contour points and the ellipsoid.

\section{Reproducibility}

To assess the reproducibility of the 3D retinal shape determination, four subjects were examined twice in two subsequent MRI sessions. The reproducibility was

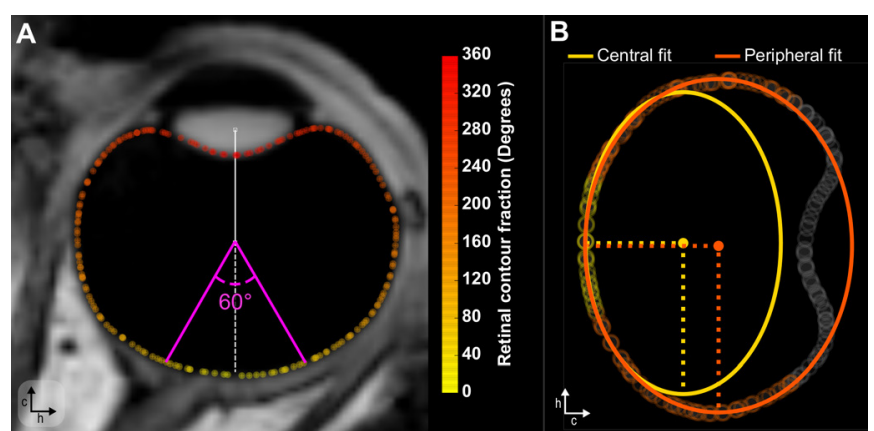

Figure 2 Quantification of the retinal shape for multiple fractions of the retinal contour. (A) The reported angles for specific retinal contour fractions are calculated with respect to the vitreous body centre. (B) Example of a 2D fit for the central and peripheral retina. 2D, two-dimensional.

evaluated per subject by comparing the parameters of the 3D fitted ellipsoids.

\section{Comparison between 3D and 2D fitting methods}

To evaluate the agreement between 3D and twodimensional (2D) retinal shape fitting, 2D ellipse fits were performed on transversal and sagittal slices of the retinal contour and combined into a 3D ellipsoid. Three different methods to define the 2D ellipse centre were evaluated. In the first method, the ellipse centre was one of the fitting parameters, similar to the 3D ellipsoid fitting method. In the second method, the ellipse centre was fixed to the vitreous body centre. In the third method, the ellipse centre was fixed at half the laser interferometry measured axial length from the posterior pole and centred on the visual axis. A complete overview of the 2D fitting procedures can be found in online supplemental appendix A. The 2D-derived ellipsoids were compared with the 3D fitted ellipsoid in terms of ellipsoidal parameters and in terms of the mean of the absolute shortest 3D distances to the MRI-based retinal contour.

\section{Considered retinal contour fraction}

The dependency of the fitted retinal shape parameters on the evaluated fraction of the retinal contour was assessed by subdividing the contour using on the angle with the vitreous body centre, and subsequently fitting 41 different fractions of the contour, ranging from the central $60^{\circ}$ to a maximum of $300^{\circ}$ in steps of $6^{\circ}$ (figure 2). Based on this analysis, the stability of the retinal shape parameters as function of retinal fraction as well as the optimal fractions to describe the central or peripheral retina were determined.

\section{RESULTS}

The 31 included subjects had a mean age of $31.1 \pm 16.2$ years $($ mean \pm SD) and a mean spherical equivalent of $-1.9 \pm 2.1$ Diopter. 21 subjects $(68 \%)$ were female. The laser interferometry measurements showed a mean internal anterior chamber depth of $3.1 \pm 0.3 \mathrm{~mm}$ and a mean axial length of $24.0 \pm 1.2 \mathrm{~mm}$. The MRI scans were successfully acquired for all subjects, and segmentation 
succeeded automatically for most subjects. Due to locally lower image contrast caused by movement artefacts, the initial guess of the retinal contour had to be manually adjusted prior to the automatic segmentation for two subjects, and the initial guess of the lens contour for one subject.

\section{Eye orientation}

The three evaluated methods to correct for gazing differences between subjects required a mean rotation of $17.3^{\circ} \pm 8.6^{\circ}$. These three evaluated methods resulted in very similar contours, with a mean distance between contours of $0.03 \pm 0.03 \mathrm{~mm}$ (5th percentile: $0.00 \mathrm{~mm}$, 95th percentile: $0.07 \mathrm{~mm}$ ). Additionally, the amount of cyclotorsion induced by the gazing correction was similar with a mean difference of $1.6^{\circ} \pm 1.9^{\circ}$. As these contour differences are within $1 / 10$ of the acquisition voxel size, and therefore, below the accuracy of the MR-data, all subsequent assessments were performed with the Euler method, which was spatially located in between the other two methods.

\section{Agreement between MRI and laser interferometry}

The distance between the centre of lens and the retina differed significantly between MRI and laser interferometry (paired-samples t-test: $\mathrm{p}<0.01$ ). The distance measured with MRI was generally shorter than the distance measured with laser interferometry, with a mean difference of $0.10 \mathrm{~mm}$ and Bland-Altman limits of agreement of -0.18 and $0.39 \mathrm{~mm}$ (figure $1 \mathrm{C}$ ).

\section{D fitting}

The 3D fitting succeeded automatically for all eyes and all fractions of the retinal contour. Overall, an increase in evaluated fraction resulted in a larger mean distance between the 3D ellipsoid and the retinal contour (figure 3). For the central retina, the fitted ellipsoid parameters showed a high variation between different

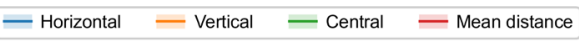

- Mean \& $95 \% \mathrm{Cl} \quad$-- Typical example

A
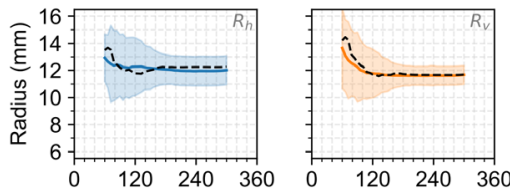

B
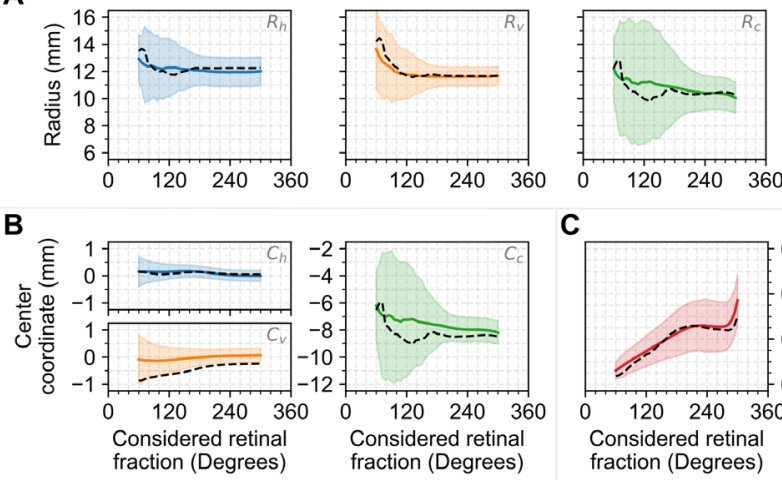

c

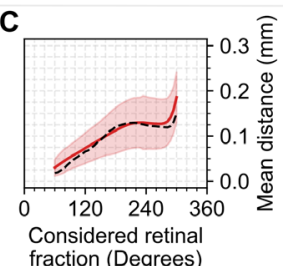

Figure $33 \mathrm{D}$ ellipsoid fitting results as function of the considered retinal contour fraction (A) The radii of the 3D ellipsoids. (B) The centre coordinates of the 3D ellipsoids. (C) The mean distance between the resulting ellipsoid and the evaluated retinal contour. 3D, three-dimensional. evaluated fractions. However, when more than the central $120^{\circ}$ of the retinal contour was evaluated, the parameters remained relatively stable, with the most stable description being achieved between $220^{\circ}$ and $280^{\circ}$ (figure 3). For fractions larger than $280^{\circ}$, the ciliary body was often included in the fit and the mean distance between the fitted ellipsoid and the measured retinal contour was relatively large (figure 3 ).

The ellipsoid radii and centre coordinates differed between evaluated retinal fractions, while the rotations remained relatively constant (online supplemental appendix B, figure B1). For $\alpha$ and $\beta, 96 \%$ of all fitted rotations were below $15^{\circ}$, with a mean of $-0.2^{\circ} \pm 5.4^{\circ}$. Additionally, $54 \%$ of all fitted ellipsoids had less than $0.5 \mathrm{~mm}$ difference between the horizontal and vertical radius. For these symmetric ellipsoids, virtually all possible angles were observed for $\gamma$. For asymmetric ellipsoids, however, $69 \%$ of all observed $\gamma$ 's showed a mean exorotation of $7.1^{\circ} \pm 21.3^{\circ}$. Additional details about these rotations can be found in online supplemental appendix B.

Overall, two different sets of ellipsoids describing the retinal shape were distinguishable for each subject. The first set describing the central retina, ranging from $60^{\circ}$ to $120^{\circ}$ of the retinal contour, and the second set describing the complete retina including the peripheral parts, ranging from $220^{\circ}$ to $280^{\circ}$ of the retinal contour. For numerical comparisons, the results for $60^{\circ}$ were used as representation of the central retina and the results for $240^{\circ}$ as representation of the peripheral retina. The primary difference between these central and peripheral results was a mean shift of the ellipsoid centre of $1.8 \mathrm{~mm}$ posteriorly when the peripheral retina was included. This shift directly affected the other ellipsoid parameters, especially the radii.

For the central retina, the mean distance between the 3D fitted ellipsoid and the considered retinal contour was $0.03 \pm 0.01 \mathrm{~mm}$. The corresponding mean ellipsoid radii were $12.9 \pm 0.9 \mathrm{~mm}$ for $R_{b}, 13.7 \pm 1.5 \mathrm{~mm}$ for $R_{v}$, and $12.2 \pm 1.2 \mathrm{~mm}$ for $R_{c}$. The ellipsoid centre was generally located within $0.7 \mathrm{~mm}$ of the central axis in the horizontal and vertical direction, with a mean axial position of $6.2 \pm 1.3 \mathrm{~mm}$ posterior of the centre of the lens (figures 3 and 4; online supplemental appendix B, table B1).

For the peripheral retina, the mean absolute distance with the retinal contour increased to $0.13 \pm 0.03 \mathrm{~mm}$. The mean axial centre of the ellipsoid, $C_{c}$, was located $8.0 \pm 0.5 \mathrm{~mm}$ posterior of the centre of the lens. Additionally, a decrease of the ellipsoid radii to $11.9 \pm 0.6 \mathrm{~mm}$ for $R_{b}$, $11.6 \pm 0.4 \mathrm{~mm}$ for $R_{v}$, and $10.4 \pm 0.7 \mathrm{~mm}$ for $R_{c}$ was observed (figures 3 and 4; online supplemental appendix $\mathrm{B}$, table B1). The absolute mean difference between $R_{b}$ and $R_{v}$ was $0.5 \pm 0.4$, and the decrease in $R_{c}$ was directly correlated with the change in $\mathrm{C}_{c}\left(\mathrm{r}^{2}: 0.98, \mathrm{p}<0.01\right)$. In 13 subjects (42\%) the difference between $R_{b}$ and $R_{v}$ was larger than $0.5 \mathrm{~mm}$. On the individual level, the radii of the fitted ellipsoids decreased in size in the majority $(91 \%)$ of the subjects between the central and peripheral retina, with only two subjects $(6 \%)$ showing an increase in all three 

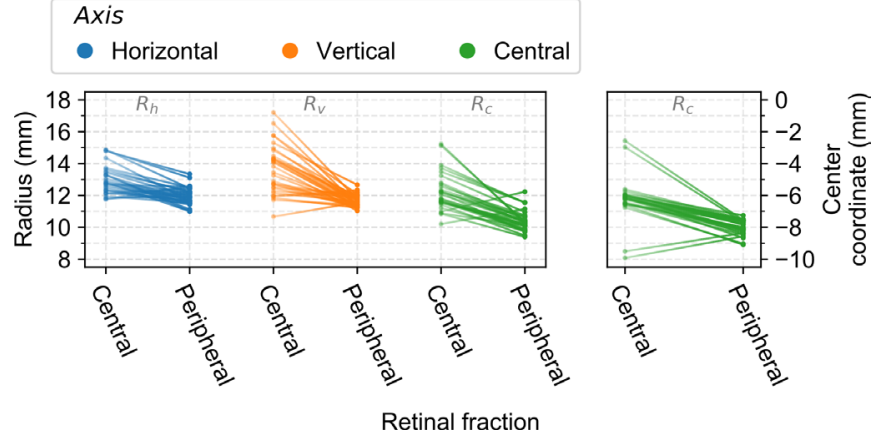

Figure 4 Differences in 3D ellipsoid parameters between the central and peripheral retina. Left: The ellipsoid radii along the horizontal (blue), vertical (orange) and central (green) axis showing a decrease in all three radii for an increase in evaluated retinal contour for the majority of the subjects. Right: Location of the ellipsoid centre along the central axis, where a lower centre-coordinate corresponds to a more posterior location in the eye. The centre-coordinates shift posteriorly for an increase in evaluated retinal contour for the majority of the subjects. 3D, three-dimensional.

radii and one subject (3\%) showing an increase of only $R_{b}$ (figure 4 ).

\section{Reproducibility}

The reproducibility analysis showed a mean difference of $0.12 \pm 0.13 \mathrm{~mm}$ between the determined retinal contours. The reproducibility of the subsequently fitted ellipsoids was dependent on the evaluated fraction of the retinal contour, with a higher reproducibility for larger evaluated fractions. For smaller fractions, up to the central $180^{\circ}$, the mean difference between the ellipsoid radii was $0.3 \pm 1.2 \mathrm{~mm}$, with a maximal difference of $5.9 \mathrm{~mm}$ (online supplemental appendix B, figure B2). For larger fractions, this mean difference was $0.0 \pm 0.1 \mathrm{~mm}$, with a maximal difference of $0.5 \mathrm{~mm}$ (online supplemental appendix B, figure B2).

\section{Comparison between 3D and 2D fitting methods}

Irrespective of the used 2D fitting method, the 3D fitting method resulted in smaller mean distances between the fit and the retinal contour (figure 5). The mean difference in centrally determined ellipsoid radii between 3D and $2 \mathrm{D}$ fitting methods was $2.9 \pm 0.9 \mathrm{~mm}$ when using the free centre $2 \mathrm{D}$ fit, $1.7 \pm 1.0 \mathrm{~mm}$ when using the vitreous body fixed 2D fit and $0.2 \pm 0.9 \mathrm{~mm}$ when using the axial length fixed 2D fit (online supplemental appendix B, figure B3), where a positive difference indicates that the measured radius is larger in $3 \mathrm{D}$ than 2D. These differences in ellipsoid radii changed to respectively $0.1 \pm 0.1 \mathrm{~mm}, 0.2 \pm 0.3 \mathrm{~mm}$ and $-0.5 \pm 1.0 \mathrm{~mm}$ for when the peripheral retina was included (figure $5 \mathrm{C}$ ).

\section{DISCUSSION}

In this study, we successfully combined high-resolution MRI scans with an automated segmentation and 3D ellipsoid fitting to describe the shape of 31 eyes. Additionally, the dependency of the resulting parametrisation on the

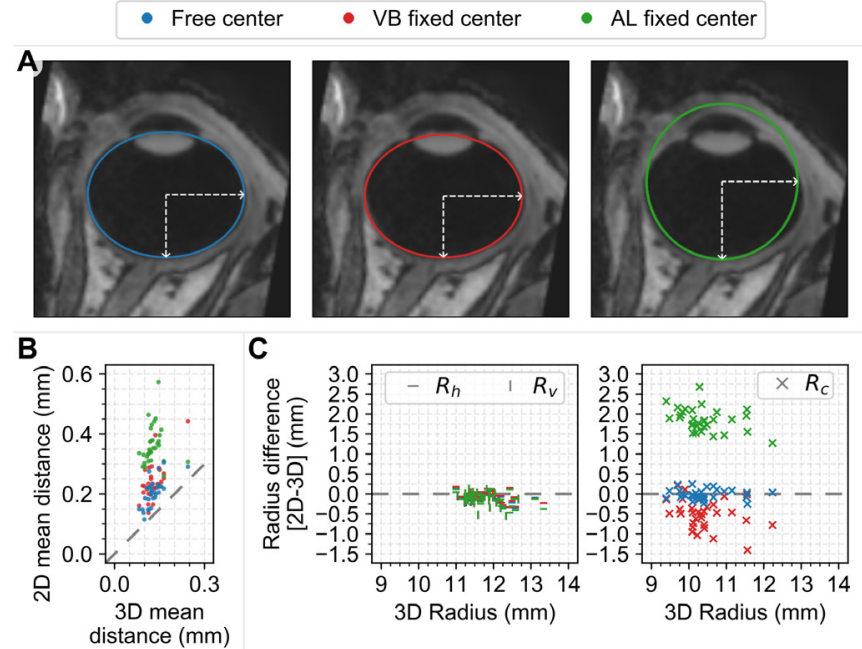

Figure 5 Comparison between 2D-derived and 3D ellipsoids fitted to the peripheral retina. Three different two 2D fitting methods are shown, one with a free centre (blue), one with a vitreous body fixed centre (red) and one with an axial length fixed centre (green). (A) Examples of the 2D fitted ellipses for a representative subject. (B) Mean distance to the original 3D contour of the ellipsoid model based on the $3 D$ and $2 D$ fits. The 3D fits consistently provided a more accurate description. (C) Differences between the 3D and 2D determined ellipsoid radii of the ellipsoids as function of the $3 \mathrm{D}$ radii. $2 \mathrm{D}$, two-dimensional; $3 \mathrm{D}$, three-dimensional.

included retinal contour and its reproducibility were evaluated. Finally, a 3D ellipsoid description based on 2D imaging data was compared with the 3D results.

The used MRI acquisition methods resulted in highresolution $3 \mathrm{D}$ images of the eye that provided a base for accurate segmentation of the eye. With mean differences of only $0.1 \mathrm{~mm}$, the segmented contours showed to be in line with laser interferometry results for the central retina. Although different methods can be used to correct these contours for differences in gazing direction during MR-imaging by aligning them to the same axis, these methods resulted in almost identical data with negligible mean differences of $0.03 \mathrm{~mm}$. Furthermore, the reproducibility of the $3 \mathrm{D}$ segmented retinal contours was high, with a mean difference of $0.12 \mathrm{~mm}$. Although these results were obtained using a high-resolution 7 Tesla MRI and a custom-made eye coil, it has recently been shown that images with a similar resolution and quality can be obtained using clinical 3 Tesla MRI-scanners and commercially available eye-coils, ${ }^{18} 28$ enabling clinical application of these methods.

The obtained MRI-derived retinal contours could be accurately described by ellipsoids, with mean differences of $0.03 \mathrm{~mm}$ for the central and $0.13 \mathrm{~mm}$ for the peripheral retina. Optically, these differences correspond to refractive differences of approximately 0.1 and 0.3 Diopter, which is sufficient to study for example the effect of peripheral vision on the progression of myopia in children. ${ }^{9}$ However, the parameters for the central retina, such as the horizontal radius, showed a 
high within-subject variability for small changes in evaluated retinal fraction, although the resulting ellipsoids accurately describe the measured contour. For these evaluations, a $1.6 \mathrm{~mm}$ decrease in the central radius $R_{c}$ can for example be compensated by $1.5 \mathrm{~mm}$ posterior shift of the ellipsoid centre (figure 3). The resulting ellipsoids, however, differ less than $0.1 \mathrm{~mm}$ over the central $60^{\circ}$, explaining the lower reproducibility and strong variation of the individual ellipsoid parameters for different included retinal fractions (online supplemental appendix B, figure B2). A similar variation was observed when the central retina would be described in terms of vertex radius of curvature and asphericity (online supplemental appendix B, figure B4). ${ }^{71216}$ When the shape of the central retina is however obtained using 3D fitting with the centre of the ellipsoid fixed to the central axis at half the axial length, stable and reproducible ellipsoidal parameters are obtained without a significant increase in fitting residuals (online supplemental appendix B, figure $\mathrm{B} 5$ and B6). This indicates that such a reduction in $\mathrm{df}$ is required for meaningful comparison of the central retinal shape between subjects.

For the peripheral retina, a reproducible and stable ellipsoid description was found between $220^{\circ}$ and $280^{\circ}$ of the retinal contour. At these fractions, the horizontal and vertical ellipsoid centre coordinates as well as the corresponding rotations remain close to zero. As a result, these parameters could be fixed in future studies, resulting in a faster fitting procedure. In $42 \%$ of the subjects, the horizontal and vertical radii differed more than $0.5 \mathrm{~mm}$. This asymmetry could be relevant for ocular proton therapy planning, which currently uses a geometric eye model in which the eye is assumed to be rotational symmetrical. ${ }^{29}$ For such applications, an ellipsoid based on two orthogonal 2D images would already be an improvement, but will still result in larger differences with the measured 3D contours than a full 3D fit. The resulting differences in ellipsoid radii are however small, generally $\leq 0.2 \mathrm{~mm}$, when the ellipse centre is fixed to the half the axial length when describing the central retina, or when this ellipse centre is included in the fitting or fixed to the centre of the vitreous body when describing the peripheral retina.

The results obtained within this study are in accordance with earlier MRI-based retinal shape studies. For the central retina, the data of the emmetropic population of Pope et alshow mean horizonal and central ellipsoid radii of approximately 12 and $11 \mathrm{~mm}$, which are similar to the results of this study. Additionally, their data show a similar large variation between subjects, for example $6 \mathrm{~mm}$ for the horizontal radius. ${ }^{716}$ For the peripheral retina, Lim et al present results for $240^{\circ}$ of the retinal contour and Pope $e t$ al evaluated a slightly larger part, $270^{\circ}$ of the retinal contour. ${ }^{1516}$ Both studies report similar horizontal and vertical radii, $11-12 \mathrm{~mm}$ and $10-11 \mathrm{~mm}$, respectively. The radii reported by Lim $e t$ al are slightly smaller than the values reported by Pope $e t a l$ and the values reported within this study. This could be explained by the difference in the MRI resolution, as a lower resolution can result in an apparent inward shift of the retina due to partial volume effects, ${ }^{30}$ resulting in smaller radii.

While the presented results are in line with earlier MR-based retinal shape research, they differ from earlier studies using laser interferometry. The mean vertex radii of curvature for the central retina determined using laser interferometry by Verkicharla et al was about $2.0 \mathrm{~mm}$ larger than the currently presented vertex radius of curvature (online supplemental appendix B, figure B4). ${ }^{12}$ Even though this difference could be the result of the unstable central fit, it might also result from the difference in used imaging methods or the limited amount of data points, $<20$, available with laser interferometry. Due to its much larger amount of data points and the availability of 3D assessments, MRI might be more reliable than laser interferometry to quantify the retinal shape. Additionally, MRI is not influenced by refraction and is not limited to assessments of the central $80^{\circ}$ of the retina or less, making it a more favourable method to measure the retinal shape.

Other methods to image the eye, such as CT and B-scan ultrasonography, are also not affected by refraction and could therefore be considered as an alternative to MRI, especially since they are generally faster to acquire. However, CT-scans expose a subject to radiation and have a lower resolution than MRI and B-scan ultrasonography is generally limited to a 2D field of view and has a high interobserver variability for geometrical measurements. ${ }^{31-33}$ As a result, both are less suitable than MRI to assess the 3D retinal shape.

It should be noted that the current results are based on a relatively small group of volunteers, and the presented parametric description of the retinal shape might therefore not hold for the entire population. However, the same methodology could directly be used to determine these metrics for a specific group of subjects. An additional concern could be that the presented method might be unable to describe pathological retinal shapes, for example on the presence of a staphyloma or intraocular mass. Although different authors have shown that the segmentation of MRI-scans can be adopted to include such ocular pathologies, ${ }^{34} 35$ these pathologies might result in an retinal shape that is not accurately described by an ellipsoid. ${ }^{36}$ Depending on the application, an approximate ellipsoidal description might still be sufficient in such instances, but using alternatives such as the complete 3D retinal contour should be considered.

In conclusion, this study provides a method to reproducibly determine and quantify the 3D retinal shape from MRI data. Two models are proposed, one which describes the complete posterior segment of the eye and an additional one for only the more central retina. Due to the high precision and reproducibility of this method, the resulting 3D shapes can be used as input in other research, such as optical ray-tracing simulations or the analysis of myopia progression. Furthermore, they can be used to improve the accuracy of the retinal shapes used in eye model-based treatment planning, which can for 
instance improve the proton therapy planning for eye tumours. With that, this study provides a complete base for widespread implementation of the 3D retinal shape as a parameter in the evaluation of the eye.

Contributors Securing funding: GPML and J-WMB. Concept and design: GPML and J-WMB. Data acquisition: J-WMB. Analysis: LvV, DPS, GPML, BCS and J-WMB. Drafting manuscript: LvV. Revising manuscript: DPS, GPML, BCS and J-WMB. Guarantor: J-WMB

Funding Supported in part by Agentschap NL (an agency of the Dutch Ministry of Economic Affairs) as part of the European Research Coordinating Agency/Natural Peripheral Vision (EUREKA/NPV) project. Supported in part by the European Society of Cataract and Refractive Surgeons.

Competing interests J-WMB received research support for the development of the used MRI techniques from Philips Healthcare as well as honoraria for an educational lecture from Ophthec.

Patient consent for publication Not applicable.

Ethics approval This study was approved by the local medical ethical comittee of the Leiden University Medical Center and registered in the CCMO-registry (registry number: NL45166.058.13).

Provenance and peer review Not commissioned; externally peer reviewed.

Data availability statement No data are available. Not applicable.

Supplemental material This content has been supplied by the author(s). It has not been vetted by BMJ Publishing Group Limited (BMJ) and may not have been peer-reviewed. Any opinions or recommendations discussed are solely those of the author(s) and are not endorsed by BMJ. BMJ disclaims all liability and responsibility arising from any reliance placed on the content. Where the content includes any translated material, BMJ does not warrant the accuracy and reliability of the translations (including but not limited to local regulations, clinical guidelines, terminology, drug names and drug dosages), and is not responsible for any error and/or omissions arising from translation and adaptation or otherwise.

Open access This is an open access article distributed in accordance with the Creative Commons Attribution Non Commercial (CC BY-NC 4.0) license, which permits others to distribute, remix, adapt, build upon this work non-commercially, and license their derivative works on different terms, provided the original work is properly cited, appropriate credit is given, any changes made indicated, and the use is non-commercial. See: http://creativecommons.org/licenses/by-nc/4.0/.

\section{ORCID iDs}

Luc van Vught http://orcid.org/0000-0001-8290-9071

Denis P Shamonin http://orcid.org/0000-0002-5614-5351

Gregorius P M Luyten http://orcid.org/0000-0003-3685-3868

Berend C Stoel http://orcid.org/0000-0002-5975-8559

Jan-Willem M Beenakker http://orcid.org/0000-0003-0479-5587

\section{REFERENCES}

1 Olsen T. Calculation of intraocular lens power: a review. Acta Ophthalmol Scand 2007;85:472-85.

2 Gokul A, Vellara HR, Patel DV. Advanced anterior segment imaging in keratoconus: a review. Clin Exp Ophthalmol 2018;46:122-32.

3 Tabernero J, Piers P, Benito A, et al. Predicting the optical performance of eyes implanted with IOLs to correct spherical aberration. Invest Ophthalmol Vis Sci 2006;47:4651-8.

4 Canovas C, Artal P. Customized eye models for determining optimized intraocular lenses power. Biomed Opt Express 2011;2:1649-62.

5 Makhotkina NY, Dugrain V, Purchase D, et al. Effect of supplementary implantation of a sulcus-fixated intraocular lens in patients with negative dysphotopsia. J Cataract Refract Surg 2018;44:209-18.

6 van Vught L, Luyten GPM, Beenakker J-WM. Distinct differences in anterior chamber configuration and peripheral aberrations in negative dysphotopsia. J Cataract Refract Surg 2020;46:1007-15.

7 Atchison DA, Pritchard N, Schmid KL, et al. Shape of the retinal surface in emmetropia and myopia. Invest Ophthalmol Vis Sci 2005;46:2698-707.

8 Fleury E, Trnková P, Erdal E, et al. Three-dimensional MRI-based treatment planning approach for non-invasive ocular proton therapy. Med Phys 2021;48:1315-26.
9 Smith MJ, Walline JJ. Controlling myopia progression in children and adolescents. Adolesc Health Med Ther 2015;6:133-40.

10 Atchison DA, Charman WN. Can partial coherence interferometry be used to determine retinal shape? Optom Vis Sci 2011;88:E601-7.

11 Verkicharla PK, Suheimat M, Pope JM, et al. Validation of a partial coherence interferometry method for estimating retinal shape. Biomed Opt Express 2015;6:3235-47.

12 Verkicharla PK, Suheimat M, Schmid KL, et al. Peripheral refraction, peripheral eye length, and retinal shape in myopia. Optom Vis Sci 2016;93:1072-8.

13 Verkicharla PK, Mathur A, Mallen EA, et al. Eye shape and retinal shape, and their relation to peripheral refraction. Ophthalmic Physiol Opt 2012;32:184-99.

14 Kuo AN, Verkicharla PK, McNabb RP, et al. Posterior eye shape measurement with retinal OCT compared to MRI. Invest Ophthalmol Vis Sci 2016;57:OCT196-203.

15 Lim LS, Matsumura S, Htoon HM, et al. MRI of posterior eye shape and its associations with myopia and ethnicity. Br J Ophthalmol 2020;104:bjophthalmol-2019-315020.

16 Pope JM, Verkicharla PK, Sepehrband F, et al. Three-dimensional MRI study of the relationship between eye dimensions, retinal shape and myopia. Biomed Opt Express 2017:8:2386-95.

17 Beenakker J-WM, Shamonin DP, Webb AG, et al. Automated retinal topographic maps measured with magnetic resonance imaging. Invest Ophthalmol Vis Sci 2015;56:1033-9.

18 Ferreira T, Grech Fonk L, Jaarsma-Coes M, et al. MRI of uveal melanoma. Cancers 2019;11:377.

19 Beenakker JWM, van Rijn GA, Luyten GPM, et al. High-resolution MRI of uveal melanoma using a microcoil phased array at 7 T. NMR Biomed 2013;26:1864-9.

20 Berkowitz BA, McDonald C, Ito Y, et al. Measuring the human retinal oxygenation response to a hyperoxic challenge using MRI: eliminating blinking artifacts and demonstrating proof of concept. Magn Reson Med 2001;46:412-6.

21 Kitslaar PH, van't Klooster R, Staring M, et al, eds. Segmentation of branching vascular structures using adaptive subdivision surface fitting2015/02. Orlando, Florida, USA.

22 Loop C. Smooth subdivision surfaces based on triangles 1987.

23 Porrill J, Ivins JP, Frisby JP. The variation of torsion with vergence and elevation. Vision Res 1999;39:3934-50.

24 Quaia C, Optican LM. Three-dimensional rotations of the eye. In: Kaufman PL, Alm A, Adler FH, eds. Adler's physiology of the eye: clinical application. 10 ed. St. Louis, MO: Mosby, 2003.

25 Tweed D, Vilis T. Geometric relations of eye position and velocity vectors during saccades. Vision Res 1990;30:111-27.

26 Maneewongvatana S, Mount DM, eds. Analysis of approximate nearest neighbor searching with clustered point sets. Data Structures, Near Neighbor Searches, and Methodology, 1999.

27 Ahn SJ, Rauh W, Oberdorfer B, eds. Least squares fitting of circle and ellipse. Mustererkennung 1998. Berlin, Heidelberg: Springer Berlin Heidelberg, 1998.

28 Niendorf T, Beenakker J-WM, Langner S, et al. Ophthalmic magnetic resonance imaging: where are we (heading to)? Curr Eye Res 2021;46:1251-70.

29 Varian Medical Systems. Planning reference guide for eclipse ocular proton planning 2007:1-286.

30 Soret M, Bacharach SL, Buvat I. Partial-volume effect in PET tumor imaging. J Nucl Med 2007;48:932-45.

31 Bhatt AB, Schefler AC, Feuer WJ, et al. Comparison of predictions made by the intraocular lens master and ultrasound biometry. Arch Ophthalmol 2008;126:929-33.

32 Haritoglou C, Neubauer AS, Herzum H, et al. Interobserver and intraobserver variability of measurements of uveal melanomas using standardised echography. Br J Ophthalmol 2002;86:1390-4.

$33 \mathrm{Char} \mathrm{DH}$, Kroll S, Stone RD, et al. Ultrasonographic measurement of uveal melanoma thickness: interobserver variability. $\mathrm{Br} J$ Ophthalmol 1990;74:183-5

34 Hassan MK, Fleury E, Shamonin D, et al. An automatic framework to create patient-specific eye models from 3D magnetic resonance images for treatment selection in patients with uveal melanoma. Adv Radiat Oncol 2021;6:100697.

35 Strijbis VIJ, de Bloeme CM, Jansen RW, et al. Multi-view convolutional neural networks for automated ocular structure and tumor segmentation in retinoblastoma. Sci Rep 2021;11:14590.

36 Ohno-Matsui K. Proposed classification of posterior staphylomas based on analyses of eye shape by three-dimensional magnetic resonance imaging and wide-field fundus imaging. Ophthalmology 2014;121:1798-809. 